Relations industrielles

Industrial Relations

\title{
"The Emerging Worker", (Equality and Conflicts in the Mass Consumption Society, par William et Margaret Westley, McGill-Queen's University Press, 1971, 155 pages.
}

\section{André Roy}

Volume 26, numéro 3, 1971

URI : https://id.erudit.org/iderudit/028258ar

DOI : https://doi.org/10.7202/028258ar

Aller au sommaire du numéro

Éditeur(s)

Département des relations industrielles de l'Université Laval

ISSN

0034-379X (imprimé)

1703-8138 (numérique)

Découvrir la revue

Citer ce compte rendu

Roy, A. (1971). Compte rendu de ["The Emerging Worker ", (Equality and Conflicts in the Mass Consumption Society, par William et Margaret Westley, McGill-Queen's University Press, 1971, 155 pages.] Relations industrielles / Industrial Relations, 26(3), 776-781. https://doi.org/10.7202/028258ar

Tous droits réservés @ C Département des relations industrielles de l'Université Laval, 1971
Ce document est protégé par la loi sur le droit d'auteur. L'utilisation des services d'Érudit (y compris la reproduction) est assujettie à sa politique d'utilisation que vous pouvez consulter en ligne.

https://apropos.erudit.org/fr/usagers/politique-dutilisation/ 
Retour des femmes sur le marché du travail après interruption d'emploi, par B.N. Seear, Paris, O.C.D.E., 1971, $154 \mathrm{pp}$.

Cet ouvrage de l'O.C.D.E. fait un excellent tour d'horizon d'une partie importante de l'activité de la femme sur le marché du travail; il s'intéresse aux femmes qui retournent sur le marché du travail après interruption d'emploi. On sait, en effet, que la main-d'oeuvre, i.e. le concept statistique de l'offre du travail, s'est accrue au cours des dernières décennies grâce principalement à l'augmentation du taux d'activité des femmes. Le phénomène revêt donc une importance économique considérable. Mais il a aussi un impact très sérieux sur d'autres aspects de la vie en société, telle par exemple la vie familiale.

On sait que les taux d'activité des femmes célibataires se rapprochent sensiblement de ceux des hommes, selon les groupes d'âge. Il n'en est pas ainsi, il va sans dire, des femmes mariées, surtout de celles qui ont de jeunes enfants. Le retour des femmes sur le marché $\mathrm{du}$ travail constitue donc un sujet fort pertinent à étudier. L'O.C.D.E. n'offre certes pas de réponses définitives à toutes les questions, mais il en explore $\mathrm{au}$ moins quelques-unes des plus importantes facettes et $y$ suggère quelques éléments de réponses.

Parmi les questions étudiées, on peut mentionner l'orientation professionnelle, l'éducation de base, le processus de formation, les attitudes et opinions. L'auteur principal de l'ouvrage, B.N. Seear, explique comment ces questions affectent d'une façon générale le retour des femmes sur le marché du travail. Plus particulièrement, il essaie de découvrir leur signification dans chacun des neuf (9) pays étudiés : Canada, Danemark, Allemagne (RF), France, Italie, Pays-Bas, Suède, Royaume-Uni et Etats-Unis. II étudie l'évolution de la situation dans chacun de ces pays, en insistant plus spécialement sur les programmes privés et publics visant à faciliter le retour en question. Le lecteur peut même trouver un tableau récapitulant la situation qui prévalait récemment dans les neuf pays mentionnés plus haut.

Nous trouvons particulièrement intéressante une étude des coûts et avantages du retour des femmes sur le marché du travail après interruption d'em- ploi. Les auteurs, Seear et Mishan, y distinguent les coûts et avantages se rapportant à la femme elle-même, à l'employeur, à l'Etat et enfin à la société toute entière. Sur le plan de l'économie, par exemple, la rentabilité des programmes relatifs au retour des femmes plus âgées sur le marché du travail dépend «de la valeur du travail accompli par les femmes, de la durée et $\mathrm{du}$ coût de la formation, du taux de non-utilisation de main-d'oeuvre, et des coûts que représente le remplacement ou l'abandon des services que les femmes auraient accomplis chez elles et au sein de leur famille si elles n'avaient pas pris un emploi rémunéré 》 (p. 150).

Somme toute, l'ouvrage de l'O.C.D.E. n'apporte rien de très original. Son principal mérite provient probablement du fait qu'il fait le point sur la situation présente de la femme qui retourne sur le marché du travail, situation qui prévaut dans neuf pays industrialisés, $y$ inclus le Canada.

Bertrand BELZILE

\section{«The Emerging Worker», (Equality} and Conflict in the Mass Consumption Society, par William et Margaret Westley, McGill-Queen's University Press, 1971, 155 pages.

Voici un ouvrage qui mérite une attention spéciale de la part de ceux qui s'intéressent aux problèmes multiples des travailleurs dans la société contemporaine.

En neuf chapitres très denses, les auteurs, après avoir passé rapidement en revue les transformations profondes de la société que sont l'accroissement général de la richesse, l'augmentation indisciplinée de la consommation, la hausse constante du niveau d'instruction des individus et la multitude des changements technologiques, font ressortir l'impact de ces facteurs sur le comportement du travailleur dans sa vie professionelle, familiale et sociale. Ces répercussions, qui consistent dans le phénomène de la mobilité tant géographique que technique, dans l'apparition de styles de vie nouveaux, dans l'établissement d'un réseau de relations différentes au sein des institutions qui l'encadrent, provoquent des tensions et des dislocations auxquelles il faut chercher des re- 
mèdes. Et, en conclusion, les auteurs d'énoncer quelques solutions concrètes.

$\mathrm{Vu}$ dans cette perspective, l'ouvrage fait penser à un triptyque où l'on voit en quelque sorte la transformation des conditions sociales dans leurs répercussions sur les attitudes personnelles et collectives des travailleurs, ce qui crée un état de déséquilibre et d'instabilité qui commandent l'effort de réflexion et d'imagination nécessaires pour le corriger.

Considérons d'abord le premier volet de ce triptyque. Quatre chapitres y sont consacrés sous la forme de brèves analyses sociologiques portant sur l'abondance, la société de consommation, le développement de l'éducation et les changements technologiques. Sur ces thèmes, les auteurs s'en tiennent à l'essentiel - et cela donne à l'ouvrage dès le départ sa densité —, mais ils le font toujours en ayant toujours présent à l'esprit le type nouveau de travailleurs qui en sortira.

Du chapitre consacré à l'abondance, qu'il suffise de retenir quelques observations-clés. Il s'agit d'une société fortement urbanisée où la majorité des gens disposent de revenus leur permettant de se procurer, outre la nourriture, le vêtement et le logis, un éventail plus ou moins grand de biens durables, de biens de civilisation, société donc où la part du revenu consacrée à l'alimentation devient de plus en plus faible, où de moins en moins de gens s'adonnent à l'agriculture, où il y a accumulation de capitaux, où le niveau d'instruction est élevé, bref une société qui tend vers l'égalisation des revenus.

Mais il y a une ombre au tableau. La pauvreté est toujours là, même si elle est refoulée dans certains quartiers des grandes villes ou dans des régions rurales sous-développées. Et cette pauvreté, elle est d'autant plus cruelle, d'autant plus dure à supporter qu'elle coudoie l'abondance. Cette situation particulière cache-t-elle quelque danger? Cette remarque frappante des auteurs laisse songeur. La pauvreté, écrivent-ils, est de tous les temps, mais «ce qui est nouveau, c'est qu'une génération a grandi qui, en majorité, en certaines parties du monde, n'a connu jusqu'ici dans sa vie active que la prospérité, le plein emploi, des revenus sans cesse croissants tout en jouissant de possibilités d'instruction sans précédent $\gg$. Aux Etats-Unis, par exemple, de 1947 à 1968, le revenu moyen de la famille américaine a triplé.

Cette opulence a donné naissance à la société de consommation, l'une servant d'étançon à l'autre. Aussi, les économistes reconnaissent-ils aujourd'hui que, pour garder l'économie sur la voie du progrès, le comportement du consommateur devient d'une importance primordiale, au moins autant que celui de l'investisseur. Mais comme l'attitude du consommateur est commandé par son optimisme ou son pessimisme selon les circonstances plutôt que par ses revenus mêmes, il s'ensuit que la prospérité générale soit remise entre les mains de la volonté de l'acheteur. L'industrie et l'Etat auront beau se débattre et pousser sur la production, c'est finalement le consommateur qui aura le dernier mot.

Dans l'ordre pratique, les auteurs relèvent trois faits qui sont de poids dans ce processus : le travail de la femme mariée, l'utilisation du crédit à la consommation et les habitudes de consommation.

Les Auteurs attachent beaucoup d'importance au travail de la femme mariée, parce que celle-ci ne cherche plus à travailler que lorsque son apport est essentiel à la subsistance de la famille, mais parce que son revenu supplémentaire est souvent celui qui, au sein de la cellule familiale, permet effectivement d'accrô̂tre le niveau de vie. De fait, aux EtatsUnis, en l'espace de vingt ans, le nombre des femmes mariées au travail a doublé.

Le crédit à la consommation jouerait un rôle identique. Sa masse s'est accrue à un rythme vertigineux. Au Canada, elle est passée de 835 millions en 1948 à plus de 7 milliards en 1965 . Le crédit à la consommation est utilisé surtout par les jeunes possédant un emploi stable dont ils escomptent retirer un revenu qui ne cessera pas.

Enfin, la société de consommation est caractérisée par le désir de tous de se procurer les mêmes biens, les mêmes satisfactions. Quels que soient l'âge, le revenu ou le groupe professionnel, les habitudes tendent à s'uniformiser. «Ouvriers et patrons possèdent et utilisent des appareils de télévision, des automobiles, des jeux d'outils de bricolage; les uns et les autres peuvent avoir ou louer une maison de villégiature et faire du ski pendant l'hiver. Au fond, les uns et les autres se procurent les mêmes objets 
enveloppés dans des emballages différents $\gg$.

En résumé, dans la société de consommation, l'état de confiance du consommateur a beaucoup de poids. Autant que l'Etat et l'industrie, il contribue à la croissance et à la stabilité de la vie économique.

L'instruction vient à son tour s'insérer dans le processus dont elle constitue en quelque sorte un des supports essentiels. Les statistiques citées ici par les auteurs permettent de voir comment les progrès de l'instruction ont été considérables au cours des trente dernières années. On est en présence, pourrait-on dire, d'une pyramide dont la base s'élargit sans cesse et dont le sommet pointe de plus en plus haut. Le phénomène est à noter: d'une part, on peut attribuer dans une bonne mesure le progrès économique à la hausse généralisée du niveau de scolarité; d'autre part, plus la société s'enrichit, plus elle tend à engager de capitaux dans l'instruction, d'où l'on peut affirmer que l'on n'est pas en présence d'une mode, d'une impulsion transitoire, mais d'un véritable phénomène structurel.

Bref, deux forces poussent ensemble vers une scolarité plus élevée : les nations, désireuses d'élever leur niveau de développement et sachant que celui-ci repose sur une formation technique et scientifique plus avancée, plus diversifiée, y voient l'instrument essentiel pour assurer l'amélioration graduelle des méthodes de production. C'est ce qu'on pourrait appeler l'instruction-investissement. Pour leur revenu personnel, ce qui est confirmé par l'observation quotidienne. Les Auteurs notent que l'on en est rendu à ce point où, monnayée, la durée de la scolarité vaut plus que le quotient intellectuel. C'est pourquoi, réagissant devant leur expérience personnelle, les parents voudront coûte que coûte que leurs enfants soient plus instruits qu'eux.

Par ailleurs, c'est l'instruction qui rendra le travailleur plus mobile, lui permettra de s'adapter aux changements technologiques et d'améliorer son statut professionnel. Mais l'instruction n'aurait qu'assez peu d'influence directe sur les habitudes de consommation, si ce n'est qu'elle peut stimuler la vente des imprimés. Toutefois, par le biais de l'accroissement du revenu qu'elle favorise, elle a tendance à élever le pouvoir d'achat.

II y a cependant une contrepartie aux avantages que peut procurer l'instruction. Plus l'homme acquiert de connaissances, plus il nourrit d'espérances: espoir d'une sécurité mieux assise, de gains plus élevés, de responsabilités professionnelles et sociales plus grandes. Ainsi, les emplois subalternes, les tâches répétitives et monotones ne plaisent guère, engendrent les sentiments d'aliénation ou inclinent à la passivité. En conséquence, observent les Auteurs, il faut s'attendre à beaucoup de mécontentement chez les travailleurs obligés d'accepter des emplois d'un intérêt moindre que ceux que leur niveau d'instruction leur permettrait d'espérer normalement. Ils signalent que des études faites au sein de certaines catégories professionnelles révèlent qu'on s'y plaint de salaires trop bas, de manque de travail intéressant, d'avancement trop lent. La satisfaction au travail diminue proportionnellement au degré du niveau d'instruction, principalement dans les groupes de collets blancs, quoique le phénomène soit beaucoup moins marqué chez les travailleurs manuels.

Cette situation nouvelle a aussi des répercussions dans la vie sociale et politique. Le syndicalisme est de moins en moins considéré comme une « œuvre», mais plutôt comme un moyen pratique de défendre les intérêts des travailleurs. Les organismes de gestion, qu'il s'agisse de l'Etat ou des entreprises privées, devenant de plus en plus spécialisés, les syndicats doivent recourir aux services d'experts, d'où les querelles acerbes qui s'élèvent au sein du mouvement syndical entre permanents et représentants de la base.

De même, dans le domaine politique, la hausse du niveau d'instruction pourra déclencher des mouvements contraires : ceux qui réussissent à s'intégrer au système auront tendance souvent à accepter des points de vue nettement conservateurs, tandis que les mécontents, les insatisfaits peuvent s'orienter vers une action politique fort radicale.

Dernier phénomène qui aura des répercussions, parfois dramatiques, sur les travailleurs de toutes catégories: l'automation ou les changements technologiques. Dans sa portée générale, les Auteurs le décrivent ainsi : "Ce qui semble caractériser l'âge de l'automation et de la consommation de masse, écrivent-ils, c'est 
un état de «fluidité » dans lequel l'homme peut s'attendre au cours de sa vie active, de changer d'emploi, d'être contraint à changer d'entourage social et géographique, d'amis, de classe sociale, de mode de vie, voire de convictions et de centre d'intérêt. Et cela non pas une fois mais plusieurs. Et il sait ausi qu'il ne peut guère prévoir ce à quoi ressemblera la vie de ses enfants. Il n'est pas besoin de remonter au moyen-âge pour juger la différence qualificative entre vivre dans de pareilles conditions et vivre dans des conditions de stabilité relative».

Nous en venons ici au deuxième volet de ce triptyque. Tous ces changements, en particulier l'avènement de la société de consommation et de la hausse générale de la scolarité, créent un environnement où la mobilité devient possible, qu'il s'agisse de mobilité professionnelle, géographique ou technique.

Les Auteurs donnent de chacune de ces formes de mobilité une définition qu'il est intéressant de noter : la mobilité professionnelle est l'habileté et le consentement à passer d'un emploi à un autre, à monter ou à descendre l'échelle des fonctions; la mobilité géographique consiste dans l'habileté et le consentement des individus à se déplacer d'un endroit à l'autre pour répondre aux occasions d'emploi ; la mobilité technique est l'aptitude à acquérir une compétence nouvelle et à s'adapter aux changements dans les processus de production.

La mobilité a pour conséquence d'entraîner des modifications dans la composition de la force ouvrière. Point n'est besoin d'insister sur ces changements qui ont été marqués par le transfert de la masse des travailleurs du secteur primaire au secteur secondaire, puis au secteur tertiaire, phénomène de mutation dont le point de départ remonte au XIXe siècle.

D’une façon générale, la mobilité joue un rôle moins important au Canada qu'aux Etats-Unis. On n'y met moins l'accent sur l'égalité ; la société y apparaît plus structurée; on s'y montre moins optimiste; on n'a moins confiance en l'avenir. Le Canada est un pays de prudence, de réserve, de contrainte. $\mathrm{Et}$ le phénomène serait encore plus marqué au Québec que dans le reste du pays. Une enquête du ministère fédéral du Travail a révélé que trente-cinq pour cent des ouvriers qualifiés, outilleurs, techniciens en électronique, etc., avaient reçu leur formation à l'étranger. D'ailleurs, les services de l'immigration s'efforcent d'attirer au Canada des ouvriers qualifiés et des professionnels.

L'avènement de la société de consommation, la hausse du niveau de l'éducation et l'automation ont aussi bouleversé le mode de vie de la population ouvrière. Il $\mathrm{y}$ a d'abord le phénomène d'urbanisation. Au début du développement de la société industrielle, les travailleurs vivaient au cœur des villes, près des usines, dans des quartiers qui leur étaient propres, alors que les banlieues étaient l'apanage des classes aisées. Qu'il suffise, pour ce qui est de chez nous, de penser au quartier Saint-Sauveur à Québec et au quartier Saint-Henri à Montréal, à la banlieue de Sillery dans la capitale et à la banlieue de Westmount dans la région métropolitaine. Depuis une vingtaine d'années, la situation a considérablement changé. Les familles ouvrières ont gagné les banlieues à leur tour où elles sont devenues propriétaires. Aux Etats-Unis, soixante-quinze pour cent des collets bleus, membres des syndicats, vivent en banlieue.

Ces changements ont eu pour résultat de modifier la nature des relations sociales des travailleurs. Ceci est surtout apparent en ce qui concerne la vie familiale. Le travailleur est beaucoup moins grégaire. Son foyer est devenu son centre d'intérêt principal, où il s'adonne à l'entretien et à l'enjolivement de sa propriété. Les relations sociales sont réduites à leur plus simple expression, c'est-à-dire à des relations de bon voisinage. Cette constatation tend, dans une certaine mesure, à contredire l'opinion générale selon laquelle la famille serait en voie d'effritement. En réalité, la famille cherche de plus en plus à se suffire à elle-même. Dans son comportement, l'ouvrier adopte de plus en plus les mœurs de la classe moyenne. Les Auteurs le notent ainsi : «Dans la famille ouvrière moderne, maris et femmes agissent en couple véritable et, s'ils ont des amis, ce sont des amis mutuels. Le mari partage ses loisirs avec sa femme. A l'intérieur de la famille, le mari participe davantage aux travaux du ménage et à l'éducation des enfants », d'où il s'ensuit que le statut de l'épouse du travailleur moderne s'est amélioré, qu'il $\mathrm{y}$ a une tendance à limiter le nombre des enfants à trois ou quatre, afin de pouvoir leur procurer ce qui a manqué aux parents. 
On observe aussi une transformation notable des mentalités dans le milieu de travail. Non seulement rencontre-t-on plus d'insatisfaction à cause de l'extrême émiettement des tâches, mais davantage encore on apprécie moins, quand on ne la déteste pas radicalement, l'atmosphère ambiante. Et ce phénomène touche encore plus le personnel de bureau que les travailleurs manuels.

Selon les Auteurs, ces faits se traduisent dans la pratique par une transformation radicale dans les relations qui s'établissent au sein des syndicats, à l'intérieur des entreprises et dans la vie de la collectivité elle-même.

Celle-ci apparaît d'abord dans les relations entre les syndicats et leurs membres. Les effectifs syndicaux ont tendance à décliner par rapport à la main-d'œuvre totale. Aux Etats-Unis, le pourcentage des syndiqués est tombé de sept pour cent au cours des dernières années par comparaison avec ce qu'il était à la fin de la guerre. On observe la même chose au Canada, quoique les collets blancs y offrent moins de résistance à la syndicalisation.

Comment cette résistance peut-elle s'expliquer? Les Auteurs en donnent plusieurs raisons. Plus instruits, les travailleurs sont de plus en plus convaincus que l'amélioration de leur niveau de vie est reliée à celui de l'accroissement de la productivité beaucoup plus qu'aux pressions syndicales; la diffusion de la société de consommation et de technologie tend à atténuer les caractères distinctifs entre travailleurs et employeurs, d'où il devient difficile pour les syndicats de persuader les travailleurs de leur utilité et de leur efficacité ; la crainte de l'automation, qui est cause de chômage, de déclassement, de retraite prématurée, a déplacé les centres d'intérêts des travailleurs devenus davantage préoccupés de la sécurité de leur emploi que de l'augmentation des salaires; changement dans la mentalité des membres des syndicats qui ressent les problèmes au niveau immédiat de leur travail et est enclin à rejeter les idéologies; augmentation du nombre des employés de bureau, partant de la main-d'œuvre féminine, qui sont réticents devant l'action syndicale, même si, graduellement, l'insécurité, la monotonie du travail, le peu d'occasions d'avancement puissent les inciter à agir collectivement.
Dans le domaine des relations de travail, il semble que les points de friction dans les négociations collectives seront sensiblement modifiés. En matière de condition d'emploi, les travailleurs cherchent à s'assurer la sécurité. Ils s'efforceront de transformer leurs occupations en professions véritables dans lesquelles ils ne vendent plus leur temps, mais leurs connaissances, leur culture générale, leurs capacités techniques, leur expérience, leur sens des responsabilités. En ce qui a trait aux salaires et aux avantages sociaux, l'enjeu se traduira par des conflits touchant la productivité et, de plus en plus, on en viendra à des accords où les majorations de salaires seront rattachées à des accroissements de la productivité (productivity bargaining).

Enfin, sur un plan plus général, dans les générations à venir, le travailleur cherchera à aligner son style de vie sur celui des classes moyennes, d'où il résultera que celui-ci sera beaucoup plus individualiste, mais en même temps, par un certain retour des choses, au plan du travail, il y aura tendance à la reconstitution de certaines formes d'intégration de disciplines professionnelles œuvrant aujourd'hui isolément, de même qu'une volonté plus forte des travailleurs à vouloir participer à l'action des corps publics : conseils municipaux, institutions scolaires, etc.

De cette évolution naît donc un type nouveau d'ouvrier dont les comportements seront bien différents de ceux du travailleur traditionnel. Ces changements ne peuvent que se refléter sur la société elle-même et l'ensemble de ses institutions.

Ils apparaissent d'abord dans l'entreprise. Autrefois, alors que le travailleur était moins instruit, qu'il manifestait moins d'indépendance, il était facile de faire jouer le système du paternaliste. La chose, d'ici peu de temps, ne sera pas aussi facile, et ceci ne pourra que provoquer une tension entre les travailleurs plus conscients de leur valeur, les employeurs, habitués à contrôler toutes les décisions relatives à la production, aux ventes, à l'établissement des politiques de rémunération.

$\mathrm{La}$ théorie des droits résiduaires, encore courante, fondée sur l'hypothèse que tous les droits reviennent à l'employeur à l'exception de ceux qui sont spécifiquement prévus aux conventions collectives de travail, devra être mise au rancart et céder la place à de nouvelles formules. 
Du côté syndical, même observation. Les syndicats, jusqu'ici, ont été assez peu démocratiques, précisément à cause de la faible participation des travailleurs. Un changement notable est en train de se produire. Ou le nouveau type d'ouvrier exigera de participer davantage à la vie syndicale, ou le syndicalisme en viendra à craquer. Le phénomène est apparent déjà dans la volonté de plus en plus marquée des travailleurs de régler leurs problèmes au niveau de la base.

On remarquera une tendance analogue dans les autres secteurs de l'activité économique, sociale et politique. Les Auteurs expriment bien ce nouvel état, lorsqu'ils écrivent: «Beaucoup de travailleurs vivent aujourd'hui dans l'aisance et une proportion de plus en plus forte de jeunes sont instruits. L'ouvrier de type nouveau apporte à de vieilles institutions des capacités nouvelles et des standards nouveaux de compétence. Il a une orientation très moderne, un sens différent de l'égo et une attitude beaucoup plus égalitaire et libérale au sujet de scs réactions et de ses responsabilités à l'égard d'autrui. Il n'accepte pas le système qui a été conçu pour le travailleur traditionnel. La fragmentation et la monotonie du travail lui apparaissent répugnantes et dépourvues de sens. Bref, pour l'ouvrier moderne, le travail n'est devenu qu'un moyen d'accroître son niveau de vie $\gg$.

En réalité, on peut dire que le travailleur est en voie d'être transfiguré par l'accroissement de la richesse et l'instruction, de telle manière qu'il veut et peut jouer un rôle différent dans son milieu de travail, dans son syndicat, dans la société. Cependant, étant donné que les institutions restent toujours adaptées à la mentalité des ouvriers traditionnels, il n'y a aucune possibilité pour l'ouvrier moderne de tenir ce rôle nouveau. Même dans les usines les plus automatisées, cette question n'est pas résolue, parce que, même si l'ouvrier se rend compte qu'il possède plus de responsabilités, il ne sent pas qu'il puisse obtenir un statut équivalent à son mérite. En conséquence, est-il frustré et mécontent, révolté quand il le peut, aliéné, s'il ne le peut pas. Et c'est ceci qui engendre les tensions qui conduisent à une détérioration considérable de la vie sociale. Pour corriger cet état de choses, quelles peuvent être les solutions valables?
Les Auteurs, dans ce qu'on peut considérer comme le troisième volet du triptyque, n'ont pas voulu élaborer. Ils n'offrent que quelques idées générales: mettre de côté ce qu'ils estiment être la philosophie désuète d'une organisation socio-économique fondée sur l'idée de rareté, alors qu'il y a surabondance de biens, pour adopter un comportement nouveau qui rejette les notions de concurrence et de consommation; faciliter aux travailleurs l'accès à la vraie liberté et à l'exercice de la responsabilité dont on sait que les applications concrètes à tout niveau peuvent être quasi infinies. Ces deux seuls faits, et il y en a combien d'autres, ouvrent très grande la porte à ceux qui sont encore capables d'observation, d'imagination créatrice.

《The Emerging Worker» n'est pas qu'un ouvrage instructif décrivant de très près une situation sociale en voie d'évolution rapide dans les pays qui ent atteint le stade de la société d'abondance. Les Auteurs ont beaucoup lu, beaucoup étudié, beaucoup observé. C'est pourquoi pareille étude s'impose comme sujet de méditation à ceux qui sont engagés dans l'action collective, y assumant à un degré quelconque des responsabilités de gouvernement et de direction.

\section{André ROY}

\section{The Unemployed : A Social-Psychological} Portrait, by D.W. Tiffany, J.R. Cowan and P.M. Tiffany, New Jersey, Prentice-Hall Inc., 1970, 180 pp.

Voici un ouvrage qui envisage le problème du chômage d'une façon entièrement différente de celles qu'on connaît habituellement. Au lieu de s'attarder à l'examen de l'aspect économique des sans-emplois, les Auteurs ont choisi de considérer les aspects psychologiques de ce problème. Le but principal de cet ouvrage est d'étudier les caractéristiques socio-psychologiques qui, reliées avec les obstacles de l'environnement, produisent le chômage.

En guise de conclusion principale, les Auteurs croient qu'on devrait traiter le chômage à la fois sous ses aspects macroéconomiques et, avec autant d'importance, sous ses aspects psychologiques.

Il ne s'agit aucunement d'une étude de cas. En fait, ce livre n'est qu'un résumé de la littérature sur le sujet. Afin d'illustrer les principes qu'ils émettent, les 